\title{
A delay of argument gradient in the instrumental conditioning of attitudes'
}

ROBERT FRANK WEISS

QUEENS COLLEGE OF THE CITY UNIVERSITY OF NEW YORK

Delay of argument effects in persuasive communication are analogous to delay of reinforcement effects in conditioning. Speed (1/latency) of agreement is a negatively accelerated decreasing function of delay of argument. Delay combines multiplicatively with number of persuasion trials to determine speed.

In terms of the empirical law of effect, an event which follows a response and increases the strength of that response on the next trial is called a reinforcer. In this experiment Ss read aloud persuasive communications designed so that $\mathrm{S}$ says the opinion to be learned, followed by an opinion-supporting argument. (The argument consists of information supporting the opinion, and specifically excludes repetitions of the opinion.) It seems reasonable to expect that an opinion which is followed by a convincing argument will be strengthened more than an unsupported opinion. Such an argument would then function as a reinforcer of the opinion response and might perhaps exhibit other functional properties of reinforcers. One such property is the inverse relationship between delay of reinforcement and response strength, and a logical development of the paradigm outlined above indicates that the delay of argument (time interval between the opinion response and the reinforcing argument) may be regarded as analogous to delay of reinforcement. Research in instrumental conditioning (e.g., Perin, 1943; Logan, 1960) indicates that conditioned response strength (as measured by response speed) is a negatively accelerated decreasing function of delay of reinforcement. Theoretically, then, attitude strength (as measured by response speed) should be a negatively accelerated decreasing function of delay of argument (Weiss, 1962).

\section{Method and Procedure}

The "instrumental conditioning" procedure of Weiss et al (1963) was employed. In order to study attitude "conditioning" rather than "habit reversal" all Ss selected had no initial opinion, as measured by a questionnaire 2-8 weeks before the experiment.

Under the impression that he was participating in a study of "speech patterns and decision making," each $S$ twice read the communication (and three other passages) into a tape recorder. The persuasive communication consisted of two passages; the first passage ended with the opinion to be learned, and the second passage was the opinion-supporting argument. Delay of argument was varied by inserting neutral reading material between the opinion and argument passages of the persuasive communication. The mean time to read the delay material is given below (reading times were normally distributed). There were five levels of delay: $0,3.5,8.5,23.0$, and $55.5 \mathrm{sec}$, with $22 \mathrm{Ss}$ in each delay condition $(\mathrm{N}=110)$.

The attitude measuring apparatus assessed each S's speed of agreement with the opinion after $S$ had been exposed to the persuasive communication. A statement of the opinion (preceded by 14 buffers) was projected on a screen and $\mathrm{S}$ signified his agreement (if he agreed) by moving a lever toward the statement. When an opinion was projected on the screen, an electric timer automatically began to measure latency of agreement (to $.01 \mathrm{sec}$ ). When the lever was moved $1 / 4$ in., a photobeam silently stopped the timer (speed $=1 /$ latency). The timing equipment was not visible to the Ss, who did not know they were being timed. If an $S$ did not respond within $45 \mathrm{sec}$, his speed of agreement was considered to be zero. Ss who did not agree did not move the lever. ${ }^{2}$

Resulis

Probability of agreement was a negatively accelerated decreasing function of delay of argument (Fig. 1) (Chi-square $=14.56, d f=4, p<.01$ ). The powerful effect of delay on probability of agreement makes it difficult to find an adequate representation of speed data for the entire sample. Since Ss who do not agree have a speed of zero, and probability of agreement exceeds

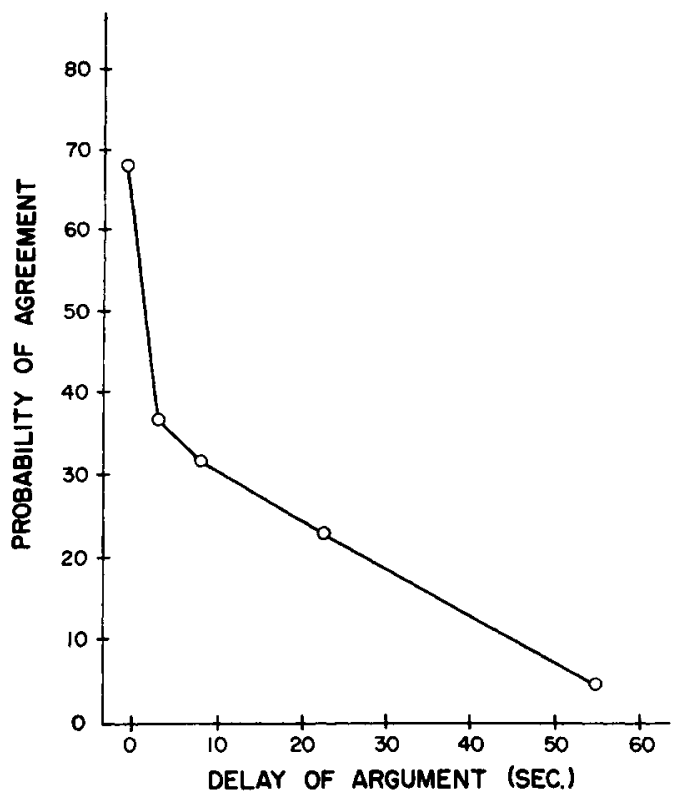

Fig. 1. Probability of agreement as a function of delay of argument. 
$50 \%$ only in the shortest delay condition, the median is very insensitive, while the mean is little more than a weighted probability score. In order to obtain a measure of delay effects on speed which would minimize these difficulties, the upper (fastest) quartile of each of the five groups $(\mathrm{N}=30)$ was examined. Median speed of agreement was a negatively accelerated decreasing function of delay of argument (Fig. 2). A Kruskal-Wallis test indicates that this effect was highly significant, even with the reduced sample size $(H=15.82, p<.005)$.

Figure 3 shows the interaction between delay (zero vs $2.8 \mathrm{sec}$ ) and number of persuasion trials (one vs two readings of the communication). The diverging curves suggest a multiplicative relationship between delay and trials. The data are assembled from the upper quartiles $(\mathrm{N}=40)$ of two exploratory experiments, run at different times, and therefore not susceptible to combined statistical treatment. Even though the long delay groups show little improvement from one to two trials, the one-trial long-delay group was significantly superior to a comparable nopersuasion control.

\section{Discussion}

The research reported here derives from a theory of attitude learning (Weiss, 1962) which is based on systematic analogies with learning research and HullMiller-Spence learning theory. The analogy between delay of argument and delay of reinforcement appears to hold in some detail. (a) In the present study of attitude "conditioning" a delay of argument gradient of the same shape as a delay of reinforcement gradient was discovered (speed measure). (b) The probability gradient also agrees with theory, although it does not afford a test over the full range of probability values; if the curve began with $100 \%$ agreement at zero delay (instead of 68\%) then the curve would be expected

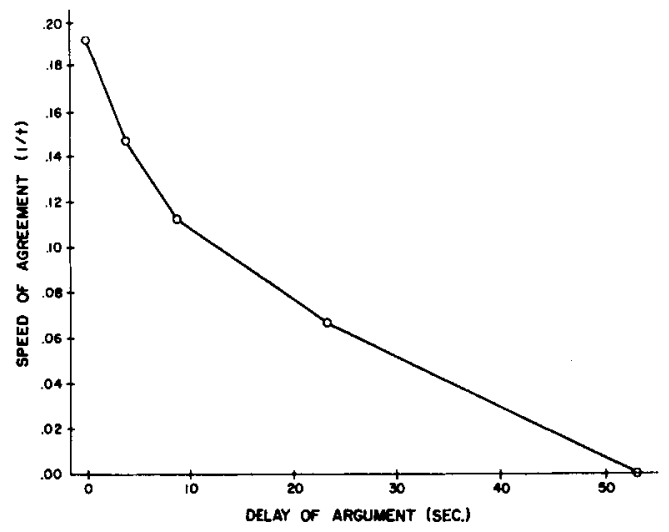

Fig. 2. Speed (1/latency) of agreement as a function of delay of argument.

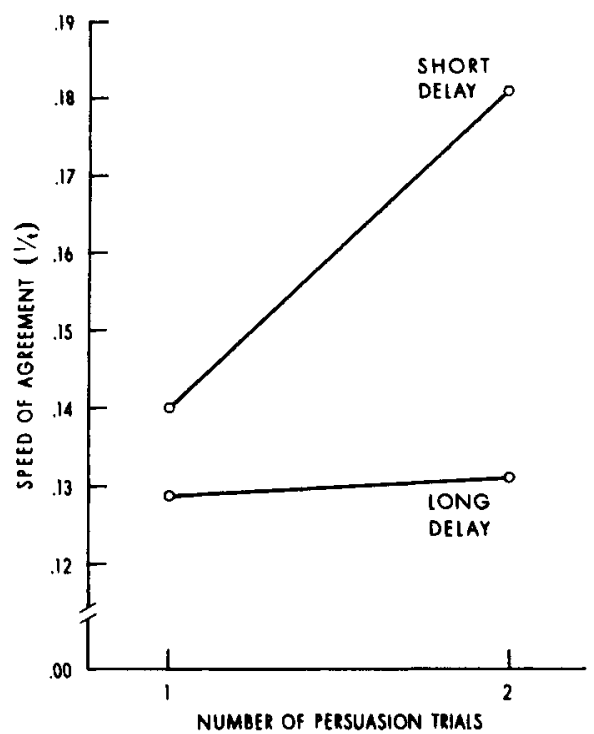

Fig. 3. Speed ( $1 /$ /atency) of agreement as a function of number of persuasion trials at two levels of delay of argument.

to exhibit a brief positive acceleration, since probability is related to excitatory potential (E) by a normal integral function (e.g., Spence, 1956). (c) Again, as in conditioning, delay appears to combine multiplicatively with the number of persuasion trials to determine speed. (d) In a previous study (Weiss et al, 1963) of the "selective learning" of attitudes, the Ss learned to choose the opinion response which had been reinforced with the shorter delay, and (e) the interaction of drive with delay was analogous to drivedelay effects in selective learning.

\section{References}

Logan, F. A. Incentive. New Haven: Xale University Press, 1960.

Perin, C. T. A quantitative investigation of the delay of reinforcement gradient. J. exp. Psychol., 1943, 32, 37-51.

Spence, K. W. Behavior theory and conditioning. New Haven: Yale University Press, 1956.

Weiss, R. F. Persuasion and the acquisition of attitudes: models from conditioning and selective learning. Psychol. Rep., 1962 , 11, 709-732.

Weiss, R. F., Rawson, H. E., \& Pasamanick, B. Argument strength, delay of argument, and anxiety in the "conditioning" and "selective learning" of attitudes. J. abnorm. soc. Psychol., 1963, 67, 157- 165.

\section{Nores}

1. Research supported by grant MH-12402, National Institute of Mental Heal th.

2. Following the typical animal conditioning procedure meant that the time from the end of the response (rather than the reinforcement) to the response-measurement was $55 \mathrm{sec}$ longer for the $55 \mathrm{sec}$ delay group than for the 0-delay group. Since $15 \mathrm{~min}$ elapsed between the second reading of the opinion response and testing on the 15 th slide, it was not expected that the $55 \mathrm{sec}$ difference would result in differential forgetting of the opinion, and a control experiment confirmed this. 